\title{
Desconocimiento como factor de riesgo para contraer COVID-19: ¿A qué nos enfrentamos?
}

\author{
Lack of knowledge as a risk factor for contracting COVID-19: What are we facing? \\ Deyanira Camacho-Osornio ${ }^{a}$ Limary Franco-Escobar $^{b}$, Alexis García-De Los Santos ${ }^{c}$, Lidia \\ Pérez-Cordova ${ }^{d}$, Dayana Rangel ${ }^{e}$, Rodrigo Sales-Bustillos ${ }^{f}$
}

\begin{abstract}
:
The objective of this study was to identify the risk factors in the socioeconomic environment, which makes us as a university community, to develop infection by SARS-CoV2. A 20-item tool assessed participants' knowledge of COVID-19. Questions related to age, gender, socioeconomic status, educational level, place of origin, religion, source of information used, and preventive measures against COVID-19 were included. The average age of the participants were 25.1 years, $62.5 \%$ being female and $37.5 \%$ male, 34 participants practicing Catholicism and 4 Christianity and its variants. A third belong to an urban locality and a minority of $3 \%$ belong to a rural locality, $55 \%$, of the households of families do not have studies. Scientific dissemination texts predominate as a source of information, 23 people have left their home 1-3 times. In the event of going out into the street, 32 people responded that they use face masks, they all have the resources to purchase one. In public places $96.9 \%$ maintain a healthy distance, 18 people had contact with a suspect or confirmed patient with COVID-19 infection. Distinguishing between misinformation and mistrust driven by inequality and moving away from the language of "conspiracy beliefs" can help avoid pushing people to endorse misinformation, as well as disseminating appropriate prevention measures and informing about the risk factors of COVID-19 to nearby communities to reduce the risk of infection.
\end{abstract}

Keywords:

Misinformation, COVID 19, socioeconomic status, risk factor

\section{Resumen:}

El objetivo de este estudio fue identificar los factores de riesgo en el ámbito socioeconómico, que nos hacen propensos como comunidad universitaria, para desarrollar infección por el SARS-CoV2. Una herramienta de 20 ítems evaluó el conocimiento de los participantes sobre COVID-19. se incluyeron preguntas relacionadas con la edad, genero, el estatus socioeconómico, nivel educativo, lugar de origen, religión, fuente de información utilizada y las medidas preventivas ante COVID-19. La edad promedio de los participantes fue de 25.1 años, siendo el $62.5 \%$ género femenino y $37.5 \%$ de género masculino, 34 participantes practican el catolicismo, 4 el cristianismo y sus variantes. Un tercio pertenecen a una localidad urbana y una minoría de $3 \%$ pertenece a una localidad rural, el 55\%, de los jefes de familia no tienen estudios. Los textos de divulgación científica predominan como fuente de información, 23 personas han salido de su casa de 1-3 veces, En caso de salir a la calle 32 personas respondieron que utilizan

a Autor de Correspondencia. Deyanira Camacho-Osornio, Universidad Autónoma del Estado de Hidalgo, https://orcid.org/0000-0002-91523581, Email: ca314857@uaeh.edu.mx

b Limary Franco-Escobar, Universidad Autónoma del Estado de Hidalgo, https://orcid.org/0000-0001-8467-7833, Email: fr267389@uaeh.edu.mx

c Alexis García-De Los Santos, Universidad Autónoma del Estado de Hidalgo, https://orcid.org/0000-0003-3016-4287, Email: ga249230@uaeh.edu.mx.

d Lidia Pérez Cordova, Universidad Autónoma del Estado de Hidalgo, https://orcid.org/0000-0002-9340-3794, Email: pe259982@uaeh.edu.mx.

e Dayana Rangel, Universidad Autónoma del Estado de Hidalgo, https://orcid.org/0000-0002-5942-2510, Email: dai.rb33@ gmail.com.

f Rodrigo Sales-Bustillos, Universidad Autónoma del Estado de Hidalgo, https://orcid.org/0000-0001-6681-4021, Email: sa294177@uaeh.edu.mx. 
cubrebocas, todos cuentan con recursos para adquirirlo. En lugares públicos $96.9 \%$ mantienen la sana distancia, 18 personas tuvieron contacto con un paciente sospechoso o confirmado con infección por COVID-19. Distinguir entre la desinformación y la desconfianza impulsada por la desigualdad y alejar el lenguaje de las "creencias de conspiración" puede ayudar a evitar empujar a las personas a respaldar la desinformación, así como difundir las medidas adecuadas de prevención e informar acerca de los factores de riesgo del COVID-19 a las comunidades cercanas para disminuir los riesgos de contagio.

\section{Palabras Clave:}

Desinformación, COVID 19, nivel socioeconómico, factor de riesgo.

\section{Introducción}

Ha surgido una tremenda incertidumbre, miedo e ira con respecto a Los orígenes, tratamientos y métodos de prevención relacionados con COVID-19. Gran parte de la evidencia necesaria para informar plenamente las respuestas clínicas y de salud pública aún no está disponible, lo que hace que la COVID-19 sea especialmente vulnerable a la proliferación de desinformación y desconfianza médica, incluidas las llamadas "creencias de conspiración". La desinformación (diseminar información falsa estratégica y deliberadamente), la información errónea (información falsa, no necesariamente con intención de engañar) y la desconfianza (más que la falta de confianza; sospecha de mala intención) son fenómenos multifacéticos, con factores motivadores subyacentes heterogéneos. ${ }^{1}$

La epidemia de infección aguda del tracto respiratorio desconocida estalló primero en Wuhan, China, desde el 12 de diciembre de 2019, posiblemente relacionada con un mercado de mariscos. Varios estudios sugirieron que el murciélago puede ser el reservorio potencial de SARS-CoV-2 puesto que los murciélagos son el reservorio natural de una amplia variedad de CoV, incluidos los virus de tipo SARS-CoV y MERS-CoV.

La transmisión de SARS-CoV-2 de persona a persona se produce principalmente entre miembros de la familia, incluidos familiares y amigos que contactaron íntimamente con pacientes o con portadores durante el periodo de incubación. ${ }^{2}$ La capacidad de transmisión rápida de persona a persona de la infección por COVID19, especialmente a través aerosoles de individuos infectados asintomáticos ha paralizado la vida en todo el mundo.

Aunque la infección por COVID-19 afecta principalmente la salud física, también puede afectar la salud mental a través del miedo a la transmisión de fuentes desconocidas y la alta tasa de mortalidad. Se considera necesario que se presten servicios oportunos y efectivos a las poblaciones vulnerables. Controlar la propagación y la transmisión de la infección es uno de los principales problemas que las autoridades están considerando con gran atención.
La Organización Mundial de la Salud (OMS) proporciona recomendaciones para las medidas de protección básicas para que el público controle la infección por COVID-19, Para que sea un socio responsable en los esfuerzos para combatir la propagación del coronavirus, deben ser conscientes del brote y estar informados sobre su papel en el control de la propagación. $^{3}$

La información errónea, la incomprensión y la falta de conciencia entre las personas pueden llevar al incumplimiento del saneamiento básico y la desobediencia de las directrices nacionales, lo que puede facilitar la transmisión rápida de la infección en la comunidad. Por lo tanto, para la implementación efectiva de medidas preventivas, es importante examinar el nivel de conocimiento y percepción de la precaución de seguridad universal, En un estudio hecho entre la población nepalesa durante esta crisis de salud global, se desarrolló un cuestionario, la encuesta en línea se organizó en tres secciones diferentes: (1) información sociodemográfica, (2)conocimiento sobre COVID-19 y (3) percepción hacia las precauciones de seguridad universales de COVID-19. Los resultados fueron los siguientes; los participantes conocían los tres síntomas principales de COVID-19 (es decir, fiebre, tos seca y falta de aliento). La mayoría también tenía conocimiento correcto sobre el período de incubación (es decir, 2-14 días), una proporción considerable no conocía el concepto de contactos cercanos (66.5\%), distanciamiento social (51\%), aislamiento (79.9\%) y el Período de autoaislamiento de 2 semanas después de la exposición a una persona sospechosa de COVID-19 (88.7\%). Además, casi la mitad de los participantes carecían de conocimiento sobre el concepto de cuarentena y la distancia ideal de dos metros durante el distanciamiento social. ${ }^{3}$

Recientemente ha habido un aumento global en la difusión de información errónea que ha afectado a la comunidad científica y al público. La crisis de salud pública que surge debido al coronavirus (COVID-19) también está comenzando a sentir los efectos de la desinformación. El personal no médico y la comunidad científica deben ser conscientes de la calidad de la información que leen y producen, respectivamente. La información errónea sobre 
la crisis de salud del coronavirus de Wuhan fue producida por los medios de comunicación, y la información errónea fue obtenida por los usuarios de Internet. ${ }^{4} \mathrm{El}$ uso de internet tiene un riesgo para la salud pública y, en casos como este, los gobiernos deberían desarrollar estrategias para regular la información de salud en internet sin censurar a la población. Dos formas principales de retroceso contra la evidencia científica dominante se han vuelto prominentes durante la pandemia por COVID-19: 1 desinformación propagada a nivel del gobierno institucional / federal para preservar el poder y socavar a los grupos ya marginados, y (2) desconfianza impulsada por la desigualdad entre comunidades que se han vuelto vulnerables por las inequidades estructurales históricas y actuales. $^{5}$

Las medidas de prevención ante el coronavirus se toman dependiendo del nivel científico con el que se disponga, en un inicio la información con que se contaba era muy poca, muy general y no había suficientes fuentes de información de donde basarnos, en este momento la información fluye de todos lados tanto que ahora existe sobreexposición informativa. ${ }^{6}$ Las fuentes de información con que la población cuenta tienen que ver con el nivel socioeconómico que es una medida total que combina la parte económica y sociológica de la preparación laboral de una persona y de la posición económica y social individual o familiar en relación a otras personas. Según Gottfried, 1985 y Hauser, 1994 incluye tres aspectos básicos: los ingresos económicos, nivel educativo y ocupación de los padres. Diversos estudios han documentado con evidencias, la relación entre el nivel socioeconómico y el estado de la salud, tanto en países y regiones, como en familias y personas. ${ }^{7}$

La dimensión demográfica la componen variables asociadas con las características de la población que, por las características de la infección por el virus SARS-CoV2 pueden ser factores que aumentan la vulnerabilidad. Esta dimensión considera también las características socioculturales de la población que dificultan el acceso a los recursos de información para prevenir el contagio, y factores que se han asociado a una menor accesibilidad a los servicios médicos indispensables una vez que han adquirido la infección. El índice de vulnerabilidad está clasificado en cuatro grados: Medio, Alto, Muy Alto y Crítico. El profesor Jeff Levin, de la Universidad de Baylor, examina el papel de la comunidad de fe en la respuesta al brote de SARS-CoV-2, ya sea como parte de la solución o parte del problema (enfatizando en la violación de las medidas de prevención por prevalecer la religión), y proporciona información sobre la pandemia de COVID-19, presentando su punto de vista como epidemiólogo que está bien familiarizado con esta situación. ${ }^{8}$ La capacidad crítica es una herramienta muy eficaz para el creyente. Gracias a un criterio formado, un creyente puede discernir entre la verdad y la mentira, descubrir el engaño, evitar el dogmatismo y el fanatismo. ${ }^{9}$ Con un criterio formado, aquella persona que profese una fe religiosa será capaz de evitar la cerrazón sobre sí mismo y podrá abrirse al respeto y a la tolerancia, de esta forma será posible convivir pacíficamente con aquellos que no comparten sus creencias. ${ }^{10}$ A medida que la pandemia de covid-19 se acelera, los gobiernos advierten a las personas con alto riesgo que sean particularmente estrictas al observar las medidas de distanciamiento social porque si se enferman tienen más probabilidades de necesitar cuidados críticos, incluida ventilación artificial y la muerte. ${ }^{11}$

Las desigualdades en el acceso a los recursos afectan no solo a quién está en mayor riesgo de infección a desarrollar síntomas o a sucumbir a la enfermedad, sino también a quién puede adoptar recomendaciones para retrasar la propagación de la enfermedad..$^{12}$ El coronavirus 2 del síndrome respiratorio agudo severo (SARS-CoV-2) se está extendiendo rápidamente por todo el mundo para causar miles de muertes cada día. Las malas respuestas de las autoridades a la propagación de la infección, la falta de medidas efectivas para la prevención, la falta de disponibilidad de opciones de tratamiento prometedoras y suficientes opciones de diagnóstico han creado una situación alarmante para el mundo. Debido a la propagación asintomática del SARS-CoV-2, desarrollar medidas de control y prevención es un desafío. ${ }^{13}$

\section{Materiales y Métodos}

Se desarrolló un trabajo de investigación mediante una herramienta de 20 ítems que evaluó el conocimiento de los participantes sobre COVID-19. Estos ítems incluyeron preguntas relacionadas con la edad, genero, el estatus socioeconómico, nivel educativo, lugar de origen (urbano o rural), religión, fuente de información sobre COVID-19 utilizado por los participantes y las medidas preventivas ante COVID-19. Participaron 40 miembros de la comunidad universitaria de distintas edades y género, respondiendo el formulario de Google forms mediante un link que les fue compartido vía whats app. El objetivo de dicha encuesta fue determinar las variables que condicionan el desconocimiento de las medidas preventivas de COVID-19.

\section{Resultados}

Se obtuvo como resultado de la encuesta que la edad de los participantes fue de entre 23 hasta los 42 años, obteniendo un promedio de edad de 25.1 años, siendo el $62.5 \%$ género femenino contra un $37.5 \%$ de género masculino de los cuales 34 participantes practican el catolicismo, 4 el cristianismo y sus variantes y 2 no 
profesan religión. Una tercera parte de la comunidad pertenecen a una localidad urbana de más de 100,000 habitantes y menos de 500,000. Así mismo, una minoría de aproximadamente $3 \%$ pertenece a una localidad rural de menos de 2,000 habitantes.
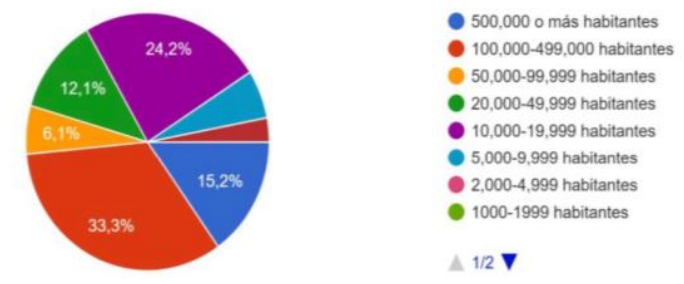

Figura 1. Número de habitantes existentes en las localidades de los participantes.

Encuestamos sobre el nivel de estudios de los participantes, obteniendo que el $55 \%$ de los jefes de familia no tienen estudios, su nivel más alto fue con preparatoria completa un $15 \%$, lo que significa que un $0 \%$ de los jefes de familia concluyeron una educación profesional completa. Sin embargo, promediando el nivel de estudios del resto de los integrantes de la familia predomina la licenciatura con un $67.5 \%$ (27 individuos), bachillerato $30 \%$ (12 individuos) y postgrado $2.5 \%$ (1 individuo).
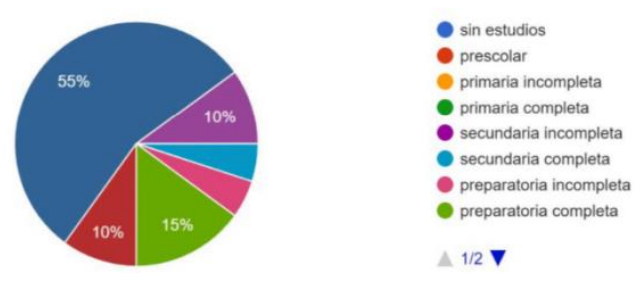

Figura 2. Nivel de estudios alcanzados por el jefe de familia.

En cuanto la información obtenida acerca de casa habitación encontramos que el $2.5 \%$ no cuenta con ningún baño completo, el $47.5 \%$ cuenta con 1 solo baño completo, mientras que el $50 \%$ cuenta con 2 o más baños completos. El número de automóviles o camionetas con que cuentan por familia reporto que el $10 \%$ no tienen ninguno, el $30 \%$ cuentan con un automóvil y el $60 \%$ con 2 o más automóviles por familia. El $95 \%$ de las familias cuentan con acceso fijo a internet, mientras que el $5 \%$ no cuentan con acceso a internet. Los textos de divulgación científica predominan como fuente de información en nuestra comunidad universitaria, las redes sociales se encuentran en segundo lugar, a pesar de no ser confiables, anteponiéndose a medios de comunicación y la opinión médica.
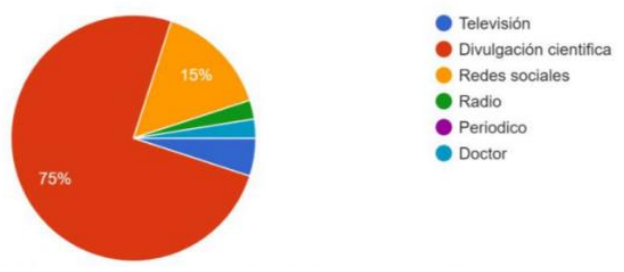

Figura 3. Fuentes de información de la comunidad universitaria.

23 personas han salido de su casa de 1-3 veces, 14 personas han salido más de 6 veces y 6 de 4 a 6 veces. (Figura 4). $53.1 \%$ de las personas han salido a la calle por necesidad, $46.9 \%$ han permanecido en casa, de las cuales las personas mayores de 14 años que trabajaron en el último mes fueron; el $7.5 \%$ ninguno trabajo, el $30 \%$ 1 persona trabajo, el $37.5 \%$ trabajaron 2 personas, $17.4 \%$ trabajaron 3 personas y el $7.5 \%$ trabajaron 4 o más personas durante el último mes. En caso de salir a la calle 32 personas respondieron que utilizan cubrebocas y todas las personas cuentan con recursos para adquirir cubrebocas.
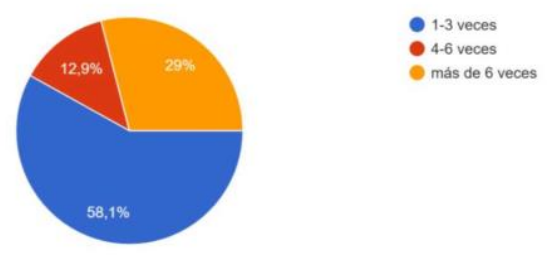

Figura 4. Frecuencia con la que realizaron actividades fuera de la casa.

Con el restante el $83.3 \%$ no utilizan cubrebocas porque es incómodo, $16.7 \%$ no utilizan cubrebocas porque no es necesario. (Figura 5). En lugares públicos concurridos $96.9 \%$ mantienen la sana distancia, mientras que el $3.1 \%$ no mantienen la sana distancia ya que no es necesario. Al estornudar $\mathrm{y} / \mathrm{o}$ toser 42 personas respondieron que se cubrían con el antebrazo y solo 2 no se cubrían por el uso de cubrebocas.

De todos los universitarios encuestados 26 no estuvieron en contacto con pacientes COVID-19 y $18 \mathrm{si}$ estuvieron en contacto.

\section{Discusión}

Los factores de riesgo que tenemos como comunidad universitaria para contraer COVID-19, se reflejan en la 
relación con el nivel socioeconómico de los universitarios, la religión que practican y el desconocimiento de las medidas de prevención y de las fuentes de información que durante la pandemia que se han presentado sabiendo que la cantidad de información no siempre es confiable como se expuso anteriormente.
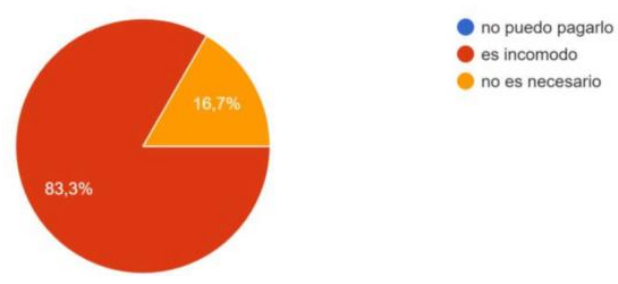

Figura 5. Uso de cubrebocas.

Nuestra muestra poblacional fue conformada por 40 miembros cuya edad promedio fue de 25 años. Dentro de los factores de riesgo con mayor peso fue él nivel socioeconómico en donde se analizaron diferentes datos como el último año de estudios del padre de familia (figura 2) en donde se observó que el $55 \%$ de los padres de familia no cuentan con ningún estudio. El número de automóviles por familia reporta que el $10 \%$ de las familias no cuentan con ningún automóvil. El acceso a internet se encuentra ligado con la fuente de información de donde obtienen datos (figura 3) se reportó que el $75 \%$ de la comunidad universitaria utiliza como fuente de información los textos de divulgación científica, sin embargo, el $15 \%$ utiliza como fuente las redes sociales lo que explica porque no siempre la información que recibimos es completamente cierta.

Además de analizar los factores de riesgo con los que se enfrenta cada uno de los universitarios se encuesto acerca de si conocen o no las medidas de seguridad para prevenir COVID-19. El lavado de manos que es una de las medidas de prevención más importantes para la prevención de COVID-19, con una duración mínima de 2040 segundos con agua y jabón cómo se han realizado las recomendaciones por la OMS el $100 \%$ de los universitarios conocen la técnica adecuada, por lo que al conocer la técnica la pondrán en práctica disminuyendo el riesgo de contraer COVID -19. En la figura 4 se explica que el $46.9 \%$ de la población si ha acatado la recomendación de permanecer en casa, mientras que el $53.1 \%$ tuvo que salir por alguna necesidad, lo que nos demuestra que el $100 \%$ de la población si acató la recomendación de permanecer en casa para disminuir el riesgo de contagio.

Otra medida de prevención que es el uso de cubre bocas en caso de salir a la calle (figura 5) toda la población universitaria sí tuvo que salir de casa lo utilizó de manera correcta. Las personas que tuvieron que salir de casa por alguna razón $96.9 \%$ mantuvieron su sana distancia como lo recomendó la OMS mientras que el $3.1 \%$ no acató la orden por qué ellos consideraban que no era necesario. El estornudo de etiqueta es llevado a cabo por el $95.5 \%$ de los universitarios quienes lo realizan de manera correcta evitando con esta acción la propagación de virus. Las personas que no acataron la orden de no salir de casa superan el $50 \%$ de la muestra, exponiéndose de esta manera más que las personas que permanecieron en su casa, así también obedecer las medidas de prevención como el uso de cubre bocas, estornudo de etiqueta, mantener la sana distancia y permanecer en casa.

\section{Conclusión}

Durante esta pandemia cada día se van descubriendo nuevas cosas para enfrentarla por lo tanto distinguir entre la desinformación y la desconfianza impulsada por la desigualdad y alejar el lenguaje de las "creencias de conspiración" puede ayudar a evitar empujar a las personas a respaldar la desinformación, así como difundir las medidas adecuadas de prevención e informar acerca de los factores de riesgo del COVID-19 a las comunidades cercanas para disminuir los riesgos de contagio.

En la encuesta realizada se demostró que gran parte de la comunidad universitaria se está informando adecuadamente ya que fundamenta sus conocimientos en artículos científicos además que la mayor parte sigue las medidas requeridas necesarias para la prevención, sin embargo, aún hay una parte que puede estar en riesgo por diversos factores como lo son; el lugar de origen rural, el acceso limitado a la información verídica, reuniones religiosas entre otras. se debe fomentar el uso de cubrebocas, así como las demás medidas preventivas para evitar más contagios.

Los profesionales médicos y de salud pública tienen la responsabilidad de comunicar la ciencia de manera efectiva, precisa y accesible, sin prejuicios, y con el entendimiento de que el racismo estructural y otras formas de opresión son las causas fundamentales de la desconfianza impulsada por la desigualdad.

\section{Recomendaciones}

Los responsables políticos deben considerar cambios regulatorios y asistencia financiera continua para apoyar a las comunidades rurales en el cuidado para personas afectadas por COVID-19 mediante diversas medidas como un fondo estatal $y / 0$ federal para mejora de insumos y equipamiento adecuado. ${ }^{14}$ Una serie de estrategias generales tomadas en conjunto pueden ayudar a los 
usuarios de internet y los medios de comunicación a confirmar la veracidad de la información y de las imágenes tales como: Buscar fuentes primarias de información; leyendo textos completos (no solo los titulares), comparar datos ofrecidos por diferentes fuentes, comprobar dónde se han publicado fotos anteriormente, buscando otras publicaciones o trabajos del mismo escritor; y verificar la fecha de publicación para proporcionar un contexto orientado y actual. Tomar como referencia a Cuba con sus medidas de acción. Cuando COVID-19 fue diagnosticado en Cuba y comenzó a extenderse, fueron lanzadas una serie de iniciativas para minimizar el impacto de rumores y desinformación en la opinión pública y apoyar el llamado de la OMS para un acceso más rápido a fuentes confiables, los usuarios pueden acceder a los informes y a las últimas novedades, noticias de la OMS y la OPS, para guiar la participación pública activa en frenar la propagación de COVID-19, incluida la orientación sobre cómo las personas pueden ayudar a cortar la cadena de transmisión al adherirse a las medidas implementadas por las autoridades sanitarias y gubernamentales. ${ }^{15}$

La práctica de la religión, el cultivo de la espiritualidad y las expresiones de fe pueden mejorar nuestra capacidad de recuperación, física y psicológicamente, y mitigar los trastornos del estado de ánimo, como la depresión y la ansiedad, que se sabe desde hace muchos años que exacerban las enfermedades e inhiben la recuperación al disminuir nuestra respuesta inmune. ${ }^{16}$ Sin embargo, cabe aclarar que la práctica de la espiritualidad no se exime de las medidas básicas preventivas contra el COVID-19; como evitar acudir a lugares concurridos, la higiene de manos, el uso de mascarilla, sana distancia; medidas que se recomienda adoptar en cada uno de sus centros espirituales y llevar acabo para así disminuir la propagación.

\section{Referencias}

[1] Mian, A., \& Khan, S. Coronavirus: the spread of misinformation.[internet].US National Library of Medicine National Institutes of Health (2020, 18 marzo). https://www.ncbi.nlm.nih.gov/pmc/articles/PMC7081539/

[2] Yang G, Qing C, Zhong H, Yuan T, Shou C, et al. The origin, transmission and clinical therapies on coronavirus disease 2019 (COVID-19) outbreak - an update on the status. Mil Med Res https://www.ncbi.nlm.nih.gov/pmc/articles/PMC7068984/

[3] Singh DR, Sunuwar DR, Karki K, Ghimire S, Shrestha N. Knowledge and Perception Towards Universal Safety Precautions During Early Phase of the COVID-19 Outbreak in Nepal. J Community Health. 2020 May $13: 1-7$

[Internet] https://www.ncbi.nlm.nih.gov/pmc/articles/PMC7220640/

[4] Jaiswal, J., LoSchiavo, C., \& Perlman, D. C. Disinformation, Misinformation and Inequality-Driven Mistrust in the Time of COVID19: Lessons Unlearned from AIDS Denialism. US National Library of Medicine National Institutes of Health. (2020, 21 mayo). [Internet] https://www.ncbi.nlm.nih.gov/pmc/articles/PMC7241063
[5] Cuan-Baltazar, J. Y., Muñoz-Perez, M. J., Robledo-Vega , C., PérezZepeda, M. F., \& Soto-Vega, E. Misinformation of COVID-19 on the Internet: Infodemiology Study. National Library of Medicine National Institutes of Health. [Internet] (2020, 9 abril) https://www.ncbi.nlm.nih.gov/pmc/articles/PMC7147328/

[6] Gottlieb, M., \& Dyer, S. (2020). Information and Disinformation: Social Media in the COVID-19 Crisis. Academic emergency medicine : official journal of the Society for Academic Emergency Medicine, 10.1111/acem.14036. https://pubmed.ncbi.nlm.nih.gov/32474977/

[7] Vera-Romero Oscar, Vera Romero Franklin(2015). Evaluation of the socioeconomic status: presentation of a scale adapted in a population from Lambayeque. Rev. cuerpo med HNAAA(6)1http://sisbib.unmsm.edu.pe/bvrevistas/cuerpomedicohna aa/v6n1_2013/pdf/a09v6n1.pd

[8] Vera-Romero Oscar, Vera Romero Franklin(2015). Evaluation of the socioeconomic status: presentation of a scale adapted in a population from Lambayeque. Rev. cuerpo med HNAAA(6)1http://sisbib.unmsm.edu.pe/bvrevistas/cuerpomedicohna aa/v6n1_2013/pdf/a09v6n1.pd

[9] Muñoz Acosta, manolo , el pensamiento crítico y las creencias religiosas, Sophia, Colección de Filosofía de la Educación, núm. 24, 2018,Universidad Politécnica Salesiana[internet] [ citado el 17 junio 2020] http://dx.doi.org/10.17163/soph.n24.2018.06

[10] Hart, C.W., Koenig, H.G. Religion and Health During the COVID-19 Pandemic. J Relig Health 59, 1141-1143 (2020)[ internet]. https://doi.org/10.1007/s10943-020-01042-3

[11] Khan S, Liu J, Xue M. Transmission of SARS-CoV-2, Required Developments in Research and Associated Public Health Concerns. Front Med (Lausanne). 2020 Jun 9;7:310-318. https://www.ncbi.nlm.nih.gov/pmc/articles/PMC7295900/

[12] Bavel, J.J.V., Baicker, K., Boggio, P.S. et al. Using social and behavioural science to support COVID-19 pandemic response. Nat Hum Behav 4, 460-471 (2020) .https://doi.org/10.1038/s41562-0200884-z

[13] Lescure F, Bouadma L, Nguyen D, Parisey M, Wicky P, Behillil S et al. Clinical and virological data of the first cases of COVID-19 in Europe: a case series [Internet]. PUBMED. 2020 [cited 3 May 2020]. Available from: https://pubmed.ncbi.nlm.nih.gov/32224310/

[14] Kaufman B, Whitaker R, Pink G, Holmes G. Half of Rural Residents at High Risk of Serious Illness Due to COVID-19, Creating Stress on Rural Hospitals [Internet]. Pubmed.gov. 2020 https://pubmed.ncbi.nlm.nih.gov/32603030/

[15] Alonso-Galbán, P., \& Alemañy-Castilla, C. (2020). Curbing Misinformation and Disinformation in the COVID-19 Era: A View from Cuba. MEDICC review, 22(2), 45-46. [Internet] [ 23 junio 2020] https://pubmed.ncbi.nlm.nih.gov/32478708/

[16] Levin J. The Faith Community and the SARS-CoV-2 Outbreak: Part of the Problem or Part of the Solution? [Internet]. US National. Library of Medicine National Institutes of Health. 2020 https://www.ncbi.nlm.nih.gov/pmc/articles/PMC7265665/ 